\title{
Tubérculo de Gerdy. Una Estructura Olvidada en la Terminología Anatómica Internacional
}

\author{
Gerdy's Tubercle. A Forgotten Structure in the International Anatomical Terminology
}

Juan Pablo Pacheco' ${ }^{1}$ \& Rodrigo Lizama Pérez²

\begin{abstract}
PACHECO, J. P. \& LIZAMA, P. R. Tubérculo de Gerdy. Una estructura olvidada en la Terminología Anatómica Internacional. Int. J. Morphol., 37(4):1305-1309, 2019.

RESUMEN: En la enseñanza de la anatomía, el uso de los epónimos es cada vez más escaso, sin embargo su uso aún es evidente incluso en la literatura actualizada, en este caso el uso del epónimo se acompaña generalmente del término correcto según la Terminología Anatómica Internacional (TAI). El denominado "Tubérculo de Gerdy" (TG) es un epónimo ampliamente usado en la literatura y la comunidad científica para denominar un reparo óseo ubicado en el cóndilo lateral de la tibia, corresponde a un referente anatómico y clínico importante para inserción de músculos, ligamentos y procedimientos medicoquirúrgicos de la rodilla. A pesar de esta amplia denominación, y en comparación con otros epónimos, la TAI no la incluye y no relaciona algún término oficial con esta estructura anatómica. El objetivo del trabajo fue revisar libros usados en la enseñanza de la anatomía y artículos científicos actualizados para ver la presencia y descripción de esta estructura a fin de proponer un término que se ajuste a los principios básicos de la Terminología Anatómica Internacional en concordancia con la tarea del Programa Federativo Internacional de Terminología Anatómica (FIPAT), organismo de la Federación Internacional de Asociaciones de Anatomistas (IFAA). El TG se describe como una estructura ubicada entre la tuberosidad de la tibia y la cabeza de la fíbula, refiriéndose a él con sinónimos como tubérculo del músculo tibial anterior, tubérculo del músculo tensor de la fascia lata, tubérculo del tracto iliotibial y tubérculo anterolateral de la tibia. En revistas morfológicas aparece en artículos actuales relacionados al ligamento anterolateral. Según la descripción de la literatura proponemos como término anatómico para esta estructura "Tuberculum anterolateralis tibiae", cuya traducción es usada como sinónimo por otros autores, ajustándose así a los principios básicos de la TAI. Pretendemos que estos antecedentes sean discutidos por los expertos que conforman el FIPAT.
\end{abstract}

PALABRAS CLAVE: Tubérculo de Gerdy; Tracto iliotibial; Músculo tibial anterior; Tubérculo anterolateral de la tibia; Ligamento anterolateral.

\section{INTRODUCCIÓN}

La Terminología anatómica (TA) es la base de la terminología médica, por lo tanto es importante que los médicos y científicos de todo el mundo utilicen el mismo término para cada estructura anatómica (Vásquez \& del Sol, 2015). La Terminología anatómica internacional (TAI), es la encargada de normalizar y estandarizar estos términos que identifican cada estructura anatómica, sin embargo su utilización aún no se ha impuesto en la literatura científica, por lo que actualmente, aún surgen inconvenientes en la denominación de estructuras entre los distintos libros para la enseñanza de la anatomía, como así también en los artículos científicos publicados sobre la materia (Fuentes et al., 2016). Una de las características del lenguaje médico es el uso habitual de epónimos o términos construidos sobre nombres propios. Sin embargo, el uso de estos, no favorece el entendimiento y ubicación de las estructuras por parte de los estudiantes y clínicos (Roa et al., 2016).
La regulación de los términos anatómicos se estableció desde 1895, con la nómina anatómica de Basilea, una terminología universal con principios básicos para la denominación de cada término anatómico. Uno de los principios básicos de la terminología anatómica es la eliminación de los epónimos, no obstante, la eponimia sigue muy arraigada en la actualidad en el lenguaje de las ciencias médicas, incluso es usada indebidamente por muchos profesores de anatomía y en revistas del campo morfológico, clínico y quirúrgico (Duque-Parra et al., 2018).

Un epónimo muy utilizado en el lenguaje de las ciencias médicas es el Tubérculo de Gerdy (TG), estructura descrita por Pierre Nicolas Gerdy, médico francés y profesor de anatomía, quien lo describió como un reparo óseo ubicado en la epífisis proximal de la tibia, específicamente en el cóndilo lateral de este hueso, sirve como un punto de refe-

\footnotetext{
${ }^{1}$ Departamento de Ciencias Morfológicas, Facultad de Medicina y Ciencia, Universidad San Sebastián, Lago Panguipulli 1390, Puerto Montt 5501842, Chile. ${ }^{2}$ Departamento de Ciencias Morfológicas, Facultad de Medicina y Ciencia, Universidad San Sebastián, General Lagos 1193 , Valdivia 5110693, Chile.
} 
rencia para diferentes situaciones clínicas, como en fracturas por avulsión y como un buen lugar para obtener injertos óseos, además de ser una referencia importante en el reciente interés por estudiar el ligamento anterolateral de la rodilla y las fibras profundas del tracto iliotibial (Somford et al., 2018), así también Donegan et al. (2015) lo describe como una referencia muy útil para los abordajes quirúrgicos de la tibia proximal y el fémur distal, siendo usualmente fácil de localizar a la palpación sobre el lado anterolateral de la tibia, ligeramente inferior al espacio articular, adicionalmente Walbron et al. (2018), mencionaron el TG como referencia estructural principal para realizar las incisiones en una nueva técnica quirúrgica para el síndrome de fricción del tracto iliotibial.

La Terminología Anatómica Internacional (TAI) presenta un índice de epónimos que identifica el número del término apropiado no epónimo (Vásquez \& del Sol), sin embargo el epónimo TG no aparece en esta lista, y el nombre "Gerdy" sólo se menciona relacionado al "Ligamento de Gerdy" correspondiente al ligamento suspensorio de la axila A04.6.03.003 (Federative Committee on Anatomical Terminology, 1998). En consecuencia, este epónimo no presenta término asociado en la TAI.

Por lo expuesto anteriormente nuestro objetivo fue identificar la presencia y descripción de esta estructura en los libros de anatomía y publicaciones científicas, con la finalidad de proponer un término adecuado que se incorpore en la Terminología Anatómica Internacional, siguiendo las indicaciones y sugerencias de la IFAA la que se señala que los nombres de las estructuras deben tener un valor informativo, estar escritos en latín como lengua base y no deben ser usados los epónimos (Roa et al.).

\section{MATERIAL Y MÉTODO}

Se realizó una búsqueda bibliográfica, no sistemática, de los libros y artículos científicos en base de datos Medline y Scielo, usando la palabra clave: "Tubérculo de Gerdy" en castellano y en inglés. Se registró la presencia del epónimo, sinónimos utilizados y de manera complementaria se evidenció la descripción morfológica que los autores realizan de esta estructura, buscando conceptos o referencias comunes.

\section{RESULTADOS}

De los libros revisados, se encontró el epónimo TG en nueve de ellos, de los cuales cinco hacen alusión a algún sinónimo para este término (Tabla I). En relación con la descripción de la estructura, algunos autores utilizan como referencia la cara articular fibular de la tibia, la cual se encuentra lateral y posterior en el cóndilo lateral de la tibia, describiendo la ubicación del TG en situación superior y medial (Llusá et al., 2003) o anterior a esta superficie articular (García-Porrero \& Hurlé, 2005). La tuberosidad de la tibia, ubicada en el extremo proximal y anterior de este hueso, también es utilizada como punto de referencia para la descripción de este tubérculo, por ejemplo (Latarjet \& Ruiz Liard, 2004) quienes señalan que su ubicación es lateral a la tuberosidad. Por su parte Testut \& Latarjet (1972) describen el tubérculo como una elevación en el extremo de una cresta oblicua de dirección superior y lateral que nace desde la tuberosidad. o desde el margen anterior de la tibia según (Orts Llorca, 1959; Buchet \& Cuilleret, 1988). Finalmente algunos describen su ubicación simplemente en posición anterolateral en el cóndilo lateral de la tibia (Rouvière \& Delmas, 2002; Moore et al., 2010)

Otro parámetro para la descripción de esta estructura es su referencia como lugar de inserción. En este caso el TG se describe como inserción del músculo tensor de la fascia lata (Orts Llorca; Rouvière \& Delmas; Testut \& Latarjet) a través del tractoiliotibial (Latarjet \& Ruiz-Liard; Llusá et al.; Moore et al.; García-Porrero \& Hurlé; Bouchet \& Cuilleret; O`Rahilly, 2001) Así mismo, se le indica como inserción del músculo tibial anterior (Rouvière \& Delmas; Latarjet \& Ruiz Liard; Llusá et al.; Orts Llorca; Testut \& Latarjet).

De las descripciones anteriormente mencionadas, se desprenden los cuatro sinónimos empleados por algunos autores, para denominar el TG: i) "Tubérculo del músculo tibial anterior" (Latarjet \& Ruiz-Liard; Orts Llorca; Testut \& Latarjet) ii) "Tubérculo del músculo tensor de la fascia lata" (García-Porrero \& Hurlé) iii) "Tubérculo del tracto iliotibial", (Orts Llorca) y iv) "Tuberculo anterolateral de la tibia" (Moore et al.).

Así mismo la búsqueda en la base de datos de artículos científicos arrojó la presencia de este epónimo en revistas del área morfológica, cuyos artículos corresponden a estudios del ligamento anterolateral de la rodilla. En los cuales, el TG se utilizó como referencia para la inserción distal de este ligamento, ubicándolo en posición posterior y proximal al TG (Zhang et al., 2018), posterior al TG (Olewnik et al., 2018), o en posición intermedia entre la cabeza de la fíbula y el TG (Claes et al., 2013; Parker \& Smith, 2016; Brockmeyer et al., 2017; Toro-Ibarguen et al., 2017) En ninguno de estos artículos se describió el TG, así como no se evidenciaron términos sinónimos para él. 
Tabla I. Presencia del epónimo Tubérculo de Gerdy y uso de sinónimos en la literatura.

\begin{tabular}{lcc}
\hline Texto & $\begin{array}{c}\text { Uso del Epónimo } \\
\text { (Tubérculo de Gerdy) }\end{array}$ & Término sinónimo \\
\hline Bouchet \& Cuilleret (1988) & $\mathrm{Si}$ & No \\
Chatain \& Bustamante (1986) & $\mathrm{No}$ & No \\
Drake et al. (2015) & $\mathrm{No}$ & No \\
García-Porrero \& Hurlé (2005) & $\mathrm{Si}$ & Inserción del músculo tensor de la fascia lata \\
O'Rahilly (2001) & $\mathrm{Si}$ & No \\
Latarjet \& Ruiz Liard (2004) & $\mathrm{Si}$ & Tubérculo del músculo tibial anterior \\
Llusá et al. $(2003)$ & $\mathrm{Si}$ & No \\
Moore et al. (2010) & $\mathrm{Si}$ & Tubérculo antero lateral \\
Orts-Llorca (1959) & $\mathrm{Si}$ & Tubérculo del tibial anterior y Tuberositas tractus \\
Platzer (2008) & $\mathrm{No}$ & No \\
Pró (2012) & $\mathrm{No}$ & No \\
Rouvière \& Delmas (2002) & $\mathrm{Si}$ & No \\
Schunke et al. (2010) & $\mathrm{No}$ & No \\
Testut \& Latarjet (1972) & $\mathrm{Si}$ & Tubérculo del tibial anterior \\
\hline
\end{tabular}

\section{DISCUSIÓN}

Los epónimos aún forman un capítulo importante en las publicaciones oficiales de la TAI, a pesar de todos los esfuerzos de la comunidad morfológica por desarraigarlos del lenguaje médico (Vásquez \& del Sol) esto se puede explicar por el hecho que estos términos están profundamente arraigados en la historia médica, la educación y el lenguaje (Roa et al.). Sin embargo erradicar los epónimos es una tarea difícil, más aun si algunos defienden su uso argumentando que la utilización correcta de estos epónimos facilitan la comunicación a la vez que dan una perspectiva histórica del desarrollo de las ciencias (Somford et al.).

En la TAI se encuentran ausentes algunos términos correspondientes a estructuras anatómicas cuyos hallazgos no son recientes y que son cada vez más aceptados por los investigadores y por lo tanto, necesitarían una posible incorporación a la TAI (Fuentes et al.,). El TG es un ejemplo de estas estructuras lo cual hace complejo erradicarlo de los libros y publicaciones científicas, por lo que es indispensable la discusión y sanción de un término no epónimo para esta estructura al amparo de la IFAA, lo cual permitirá una correcta divulgación en la literatura científica, con la esperanza compartida y expuesta por Duque-Parra et al., quien manifiesta que las nuevas generaciones de docentes de ciencias morfológicas, apoyados por los editores de revistas, debieran aplicar la terminología anatómica internacional, y concientizar a sus estudiantes en que es más fácil aprender esta ciencia cuando se usa la lógica morfo-funcional, comparado con el aprendizaje de aquel otro lenguaje morfológico tradicional que implica un mayor esfuerzo memorístico basado en epónimos. De esta forma, podremos trabajar en uno de los desafíos de las ciencias morfológicas, el cual consiste en socializar la terminología anatómica internacional a los docentes clínicos para ir modificando los términos en la práctica diaria.

Precisamente suprimir los epónimos es uno de los principios básicos de la construcción de la TAI, por lo cual estos no deben formar parte de publicaciones o comunicaciones oficiales avaladas por la IFAA y sus organismos asociados, así como en revistas del área morfológica, amparadas por sociedades miembros de la IFFA o en cuyos comités editoriales formen parte miembros de la IFAA o la FIPAT, en este sentido al igual que lo expresado por Duque-Parra $e t$ al., encontramos específicamente el uso del epónimo TG en tres revistas morfológicas amparadas por sociedades miembro de la IFAA, Clinical Anatomy y Journal of Anatomy con 2 artículos cada una en la última década y Annals of Anatomy con 1 artículo en estos 10 años.

Otro principio básico es que los nombres de las estructuras deben tener un valor informativo, para lo cual es indispensable conocer las características de estructuras señaladas como epónimos, así como su implicancia para una correcta denominación en la TAI. En este sentido los libros que describen este tubérculo utilizan como referencia dos estructuras de la tibia, la tuberosidad de la tibia, de ubicación anterior y la cara articular para la cabeza de la fíbula, de ubicación posterolateral, quedando el TG en situación intermedia entre estas estructuras, esta disposición puede describirse como "anterolateral" en la epífisis proximal de la tibia, término congruente con el denominado ligamento 
anterolateral de la rodilla, estructura muy estudiada en la última década, precisamente de Lima et al. (2019) revisaron 53 artículos sobre este ligamento, concluyendo que cuando estaba presente, se insertaba distalmente en la tibia entre la cabeza de la fíbula y el TG, por lo cual la denominación de anterolateral para el tubérculo coincide con el sinónimo utilizado por Moore et al.

En relación a la descripción basada en el TG como zona de inserción, podemos observar que esta estructura es descrita como sitio de inserción del tracto iliotibial y del músculo tibial anterior, por lo cual no consideramos adecuados los sinónimos de Tubérculo del Tracto Iliotibial o Tubérculo del Tibial Anterior ya que cada sinónimo por sí solo, excluye al otro, lo cual dificulta el reconocimiento y localización de este reparo óseo.

Como se evidencia, a pesar que varios artículos y libros de anatomía, mencionan y describen el TG, utilizándolo como una referencia ósea importante, aún no existe un consenso entre la comunidad científica para establecer un término oficial para nombrarlo. La claridad de la estructura asociada y el término anatómico para un epónimo determinado permitirá difundir de forma universal un término oficial en desmedro del uso de los epónimos.

Basándonos en los principios básicos de la TAI, proponemos incluir en la terminología anatómica el término "Tuberculum anterolateralis tibiae", denominación que se acerca a lo propuesto por la Federación Internacional de Asociaciones de Anatomistas (IFAA) donde el término para cada estructura debe estar en latín, único idioma oficial para establecer el nombre de alguna estructura sobre el cual debe realizarse la traducción a otro idioma. La traducción de este término concuerda con el término sinónimo usado por Moore et al.

Pretendemos que esta propuesta sea discutida por los integrantes del Programa Federativo de Terminología Anatómica para futuras actualizaciones y publicaciones de la Terminología Anatómica Internacional.

PACHECO, J. P. \& LIZAMA, P. R. Gerdy's Tubercle. A forgotten structure in the International Anatomical Terminology. Int. J. Morphol., 37(4):1305-1309, 2019.

SUMMARY: In teaching anatomy, the use of eponyms is increasingly scarce. Nonetheless, eponyms remain evident in updated literature, in this case the use of the eponym is accompanied by the correct term according to the International Anatomical terminology (TAI ) Gerdy's tubercle (GT) is an eponym widely used in the literature and scientific community to name a lateral tubercle of the tibia. It is an insertion site of muscles and ligaments and an important anatomical reference in knee surgical procedures. Despite its importance, it is not included in the International Anatomical Terminology (IAT) and an official name for this structure is lacking. The aim of this work was to review classic books used in the anatomy teaching and recent scientific papers, and further, to propose an anatomical term for the Gerdy tubercle that fit IAT basic principles, in agreement with the International Federal Program of Anatomical Terminology (IFPAT), and organism that is part of the International Federation of Anatomist Associations (IFAA). The TG is described as a structure located between the tuberosity of the tibia and the head of the fibula, referring to it with synonyms such as tubercle of the anterior tibia, tubercle of the tensor fascia lata, tubercle of iliotibial tract and anterolateral tibial tubercle. In morphological journals it appears in current articles related to the anterolateral ligament. We propose as an anatomical term for the Gerdy's tubercle the name of "Tuberculum anterolteralis tibiae" its translation is used as a synonym by other authors and fits the basic principles of TAI. We hope that this information be considered by the experts that make up the FIPAT.

KEY WORDS: Gerdy's tubercle; Iliotibial tract; Tibial anterior muscle; Anterolateral tibial tubercle; Anterolateral ligament.

\section{REFERENCIAS BIBLIOGRAFICAS}

Bouchet, A. \& Cuilleret, J. Anatomía Descriptiva, Topográfica y Funcional. Tomo 1. Buenos Aires, Médica Panamericana. 1988.

Brockmeyer, M.; Höfer, D.; Schäfer, K.; Seil, R.; Becker, K.; Paulsen, F.; Kohn, D. \& Tschernig, T. The anterolateral ligament (ALL) of the kneePart of the iliotibial tract or a truly separate structure? Ann. Anat., 212:13, 2017.

Chatain, I. \& Bustamante, J. Anatomía Macroscópica, Funcional y Clínica. Ciudad de México, Sistemas Técnicos de Edición S.A. de C.V., 1986.

Claes, S.; Vereecke, E.; Maes, M.; Victor, J.; Verdonk, P. \& Bellemans, J. Anatomy of the anterolateral ligament of the knee. J. Anat., 223(4):3218, 2013.

de Lima, D. A.; Helito, C. P., Lacerda de Lima, L.; de Castro Silva, D.; Costa Cavalcante, M. L. \& Dias Leite, J. A. Anatomy of the anterolateral ligament of the knee: a systematic review. Arthroscopy, 35(2):670-81, 2019.

Donegan, D. J.; Seigerman, D. A.; Yoon, R. S. \& Liporace, F. A. Gerdy's tubercle: the lighthouse to the knee. J. Orthop. Trauma, 29(2):e51-3, 2015.

Drake, R.; Volg, W. \& Mitchel, A. Grey. Anatomía para Estudiantes. $3^{\mathrm{a}}$ ed. Barcelona, Elsevier, 2015.

Duque-Parra, J. E.; Barco-Ríos, J. \& Duque-Quintero, M. Eponymy that difficult obstacle that International Anatomical Terminology still does not overcome. Int. J. Morphol., 36(4):1206-9, 2018.

Federative Committee on Anatomical Terminology (FCAT). Terminologia Anatomica. International Anatomical Terminology. Stuttgart, Thieme, 1998.

Fuentes, R.; Ottone, N. E.; Bucchi, C. \& Cantín, M. Analysis of terms used in the literature to refer to temporomandibular joint capsule and joint ligaments. Int. J. Morphol., 34(1):342-50, 2016.

García-Porrero, J. \& Hurlé, J. Anatomía Humana. Madrid, McGraw-Hill, 2005.

Latarjet, M. \& Ruiz Liard, A. Anatomía Humana. Tomo 1. $4^{\text {a }}$ ed. Buenos Aires, Médica Panamericana, 2004. 
Llusá, M.; Meri, A. \& Ruano, D. Manual y Atlas Fotográfico del Aparato Locomotor. Madrid, Médica Panamericana, 2003.

Moore, K. L.; Dalley, A. F. \& Agur, A. M. R. Anatomía con Orientación Clínica. $6^{\mathrm{a}}$ ed. Barcelona, Walters Kluwer-Lippincott Williams \& Wilkins, 2010.

O’Rahilly, R. Anatomía de Gardner. Ciudad de México, McGraw-Hill, 2001.

Olewnik; Gonera, B.; Kurtys, K.; Podgórski, M.; Polguj, M.; Sibinski, M. \& Topol, M. The anterolateral ligament of the knee: a proposed classification system. Clin. Anat., 31(7):966-73, 2018.

Orts-Llorca, F. Anatomía Humana. Vol. 1. $2^{\mathrm{a}}$ ed. Barcelona, CientíficoMédica, 1959.

Parker, M. \& Smith, H. F. Anatomical variation in the anterolateral ligament of the knee and a new dissection technique for embalmed cadaveric specimens. Anat. Sci. Int., 93(2):177-87, 2016.

Platzer, W. Atlas de Anatomía: Con Correlación Clínica. Tomo 1. Barcelona, Médica Panamericana, 2008.

Pró, E. Anatomía Clínica. Buenos Aires, Médica Panamericana, 2012.

Roa, I.; Vásquez, B. \& Contreras, M. Eponyms persistence in Terminologia Histologica. Int. J. Morphol., 34(4):1245-52, 2016.

Rouvière, H. \& Delmas, A. Anatomía Humana: Descriptiva, Topográfica y Funcional. Tomo 3. 10a ed. Barcelona, Masson, 2002.

Schunke, M.; Schulte, E. \& Schumacher, U. Prometheus: Texto y Atlas de Anatomía. Tomo 1. 2a ed. Buenos Aires, Médica Panamericana, 2010.

Somford, M. P.; Nieuwe Weme, R. A.; Hoornenborg, D.; Wiegerinck, J. I.; van Raay, J. J. A. M.: Brouwer, R. W. \& Williams, A. Biographical background and origin of common eponymous terms in orthopedic surgery: anatomy and fractures in knee surgery. Eur. J. Orthop. Surg. Traumatol., 28(1):79-84, 2018.

Testut, L. \& Latarjet, A. Tratado de Anatomía Humana. Tomo 1. 9a ed. Barcelona, Salvat, 1972.

Toro-Ibarguen, A. N.; Pretell- Mazzini, J.; Pérez, E.; Pedrajas, I.; CanoEgea, J. M. \& Sanudo, R. J. The anterolateral ligament: A cadaveric study in fetuses. Clin. Anat., 30(5):625-34, 2017.

Vásquez, B. \& del Sol, M. Terminologia Anatomica and Terminologia Histologica. A meeting point between morphologists. Int. J. Morphol., 33(4):1585-90, 2015.

Walbron, P.; Jacquot, A.; Geoffroy, J. M.; Sirveaux, F. \& Molé, D. Iliotibial band friction syndrome: An original technique of digastric release of the iliotibial band from Gerdy's tubercle. Orthop.Traumatol. Surg. Res., 104(8):1209-13, 2018

Zhang, H.; Qiu, M.; Xu, Z.; Wang, W.; Chen, S.; Zhang, J.; Zhou, A.; Lu, K.; Huo, M. \& Jiang, D. The prevalence and morphological characteristics of the knee anterolateral ligament in a Chinese population. J. Anat., 233(2):213-21, 2018.

\author{
Dirección para correspondencia: \\ Juan Pablo Pacheco Muñoz \\ Lago Panguipulli 1390 \\ Pichi Pelluco \\ Puerto Montt \\ CHILE
}

Email: juan.pacheco@uss.cl

Recibido : 01-02-2019

Aceptado: 18-06-2019 